\title{
openheart Endothelial function and left ventricular diastolic functional reserve in type 2 diabetes mellitus
}

\author{
Melissa Leung, Victoria Phan, Dominic Y Leung
}

To cite: Leung M, Phan V, Leung DY. Endothelial function and left ventricular diastolic functional reserve in type 2 diabetes mellitus. Open Heart 2014;1:e000113 doi:10.1136/openhrt-2014000113

$\mathrm{ML}$ and VP are joint first authors.

Received 28 February 2014 Revised 30 May 2014 Accepted 15 July 2014

\section{CrossMark}

Department of Cardiology, Liverpool Hospital, University of New South Wales, Sydney, Australia

Correspondence to Dr Melissa Leung; melissa@unsw.edu.au

\section{ABSTRACT}

Background: Endothelial dysfunction is an early feature of vascular disease. Left ventricular (LV) diastolic reserve is the ability of the left ventricle to augment diastolic function with exercise and may be impaired in patients with diabetes mellitus (DM). It is unclear if endothelial dysfunction is related to impaired LV diastolic reserve and diminished exercise capacity.

Methods: 96 patients with type 2 DM and 10 controls had brachial artery reactivity testing, followed by exercise echocardiography. The brachial artery diameter was measured at rest and during reactive hyperaemia. LV diastolic reserve was measured as $\Delta \mathrm{e}^{\prime}$ with exercise and diastolic reserve index $\left(\Delta \mathrm{e}^{\prime} / \mathrm{rest}^{\prime} \mathrm{e}^{\prime}\right)$. Exercise capacity was calculated by metabolic equivalents (METs).

Results: Compared with controls, patients with DM had lower rest $e^{\prime}(7 \mathrm{vs} 9 \mathrm{~cm} / \mathrm{s}, p=0.002)$, lower $\Delta \mathrm{e}^{\prime}(1$ vs $4 \mathrm{~cm} / \mathrm{s}, \mathrm{p}=0.023$ ), lower $\Delta \mathrm{e}^{\prime} /$ rest $\mathrm{e}^{\prime}$ ( 0.20 vs 0.47 , $\mathrm{p}=0.003$ ) and reduced flow mediated dilation (FMD, 5 vs $15 \%, p<0.001)$. FMD was correlated with $\Delta e^{\prime}$ $(r=0.65, p<0.001)$, diastolic reserve index $(r=0.61$, $\mathrm{p}<0.001)$ and post-exercise septal $E / \mathrm{e}^{\prime}(\mathrm{r}=-0.50$, $p<0.001)$, but not with rest $e^{\prime}(r=0.13, p=0.177)$. FMD was an independent predictor of $\Delta \mathrm{e}^{\prime}(\beta=0.002$, $\left.p<0.001, R^{2}=0.47\right)$ and diastolic reserve index $\left(\beta=0.030, p<0.001, R^{2}=0.41\right)$. Younger age $(p<0.001)$, male gender $(p=0.014)$, lower body mass index $(p<0.001)$, lower rest $E / e^{\prime}(p=0.042)$ and higher FMD $(p=0.025)$ were independent predictors of higher METs $\left(R^{2}=0.52, p<0.001\right)$.

Conclusions: Patients with DM had impaired endothelial function and LV diastolic dysfunction. LV diastolic reserve and exercise capacity are linked to endothelial function. Targeting vascular risk factors to improve endothelial function may improve LV diastolic reserve and exercise capacity.

\section{BACKGROUND}

Endothelial dysfunction plays a pivotal role in the pathogenesis of atherosclerosis ${ }^{1}$ and has been shown to precede the development of atherosclerotic plaques in the coronary arteries. $^{2}$ It has also been demonstrated in the presence of vascular risk factors

\section{KEY MESSAGES}

What is already known about this subject?

- Endothelial dysfunction is an early feature of vascular disease. Left ventricular (LV) diastolic reserve is the ability of the left ventricle to augment diastolic function with exercise and may be impaired in patients with diabetes (DM).

What does this study add?

- DM patients had impaired endothelial function, LV diastolic dysfunction and diastolic reserve. LV diastolic reserve and exercise capacity are linked to endothelial function.

How might this impact on clinical practice?

- Targeting vascular risk factors to improve endothelial function may improve LV diastolic reserve and exercise capacity.

including smoking, hypertension, dyslipidaemia and diabetes mellitus (DM). ${ }^{34}$

Patients with DM may develop cardiomyopathy independent of traditional risk factors such as hypertension and epicardial coronary artery disease. The spectrum of myocardial dysfunction may range from isolated diastolic dysfunction in the early stage to overt systolic dysfunction. Diastolic dysfunction is usually manifested as exertional dyspnoea and impaired exercise capacity. Diastolic reserve is the ability of left ventricular (LV) filling pressures to remain normal with exercise and tachycardia. Analogous to an impaired contractile reserve in early LV systolic dysfunction, an impaired diastolic reserve may be seen in early stages of diastolic dysfunction such as that in diabetic cardiomyopathy ${ }^{5-7}$ Like systole, $\mathrm{LV}$ diastole is an active, energy requiring process and hence may be adversely affected by endothelial dysfunction. However, no studies have examined the possible link between LV diastolic dysfunction, exercise capacity and endothelial dysfunction in patients with DM. In this study, we aim to evaluate the link between endothelial function, exercise capacity and LV diastolic function reserve in patients with type $2 \mathrm{DM}$. 


\section{METHODS}

\section{Patients}

Ninety-eight patients with type $2 \mathrm{DM}$ were recruited from hospital clinics and from the local community. Type $2 \mathrm{DM}$ was diagnosed according to the American Diabetes Association criteria. ${ }^{8}$ Patients with type $1 \mathrm{DM}$, known congenital, valvular or coronary artery disease $(\mathrm{CAD})$, severe hypertension (systolic pressure $>200 \mathrm{~mm} \mathrm{Hg}$ and diastolic pressure $>120 \mathrm{~mm} \mathrm{Hg}$ at rest), left bundle branch block or rhythm other than sinus and those unable to exercise were excluded. All participants had normal resting ECG and were free of symptoms or signs of overt heart disease. All cardiac medications were continued throughout the studies with no modifications made to the patients' treatment regimen. Ten age-matched and gender-matched healthy individuals with no documented vascular disease were included as controls. All patients provided written informed consent. The study was approved by the Hospital Human Research Ethics Committee.

Clinical data collected included age, height, weight, waist and hip circumference, Medical Research Council dyspnoea scale, cardiac risk factors, duration of DM, medications and the presence of macrovascular and microvascular complications. The body mass index was calculated.

\section{Metabolic data}

For patients with DM, haemoglobin, glycated haemoglobin (HbA1c) levels, serum creatinine, estimated glomerular filtration rate (eGFR) using the modification of diet in renal disease formula, ${ }^{9}$ total cholesterol, lowdensity lipoprotein, high-density lipoprotein, triglycerides and urinary spot albumin to creatinine ratio were measured.

\section{Two-dimensional and Doppler echocardiography}

Two-dimensional (2D) transthoracic echocardiography was performed in the left lateral decubitus position with commercially available ultrasound equipment (M5S probe, Vivid E9, GE Medical Systems, Milwaukee, WI). All images were digitally stored on hard discs for offline analysis (EchoPAC, BT10, GE). A complete M-mode, 2D, colour, pulsed and continuous-wave Doppler examination was performed according to standard techniques at rest to assess chamber thickness, volumes and valvular morphology.

LV diastolic and systolic volumes were measured to calculate LV ejection fraction using the Simpson biplane method of discs in the apical four-chamber and twochamber views. Maximum and minimal left atrial volumes were measured similarly. The pulmonary artery systolic pressure was estimated from the tricuspid regurgitant jet maximum velocity. The mitral inflow pattern was interrogated with pulse wave Doppler by placing a 1-2 $\mathrm{mm}$ sample volume at the mitral leaflet tips in the apical four-chamber view. The peak velocities of early (E) and late (A) ventricular filling, and deceleration times of the $\mathrm{E}$ wave were measured. The mitral septal and lateral annulus systolic $\left(\mathrm{s}^{\prime}\right)$, early $\left(\mathrm{e}^{\prime}\right)$ and late $\left(\mathrm{a}^{\prime}\right)$ diastolic tissue velocities were measured with pulse-wave tissue Doppler. The Nyquist limit was adjusted to approximately $24 \mathrm{~cm} / \mathrm{s}$; a clear tissue signal with minimal background noise was recorded by optimising the gain and sample volume. A 2-5 mm sample volume was placed at the septal and lateral mitral annulus in the apical four-chamber view, paying careful attention to ensure proper alignment and minimise the incident angle. Three to five cardiac cycles were recorded for all 2D echocardiographic and Doppler measurements.

\section{Exercise echocardiography}

All patients underwent symptom limited exercise echocardiography on a treadmill protocol according to the patients' individual physical ability. Exercise capacity was measured as the metabolic equivalents (METs) achieved at peak exercise. The METs were calculated according to the American College of Sports Medicine Guidelines for Exercise Prescription and Testing as follows ${ }^{10}$ :

$$
\text { METs }=\frac{3.5+0.1 \times \text { speed }+\frac{1.8 \times \text { speed } \times \text { grade }}{100}}{3.5}
$$

where grade is given in per cent, and speed in metres per minute.

Heart rate and blood pressure were measured at rest and at 3 minute intervals during exercise. Two-dimensional images of the left ventricle were obtained in the apical (4 chamber, 2 chamber, long axis), parasternal long-axis and mid-ventricular short-axis views. These images were taken at rest and again immediately post-exercise.

Immediately after the acquisition of $2 \mathrm{D}$ images postexercise, the mitral $\mathrm{E}$ wave velocities and mitral septal annulus systolic $\left(\mathrm{s}^{\prime}\right)$, early $\left(\mathrm{e}^{\prime}\right)$ and late $\left(\mathrm{a}^{\prime}\right)$ diastolic tissue velocities were measured. Patients with inducible ischaemia evident by new LV segmental wall motion abnormalities were excluded from further analysis $(\mathrm{n}=2)$.

The mitral $\mathrm{E} / \mathrm{e}^{\prime}$ ratio was used to estimate $\mathrm{LV}$ filling pressures at rest and post-exercise. $\mathrm{LV}$ diastolic reserve was measured as $\Delta \mathrm{e}^{\prime}$ (septal $\mathrm{e}^{\prime}$ post-exercise- $\mathrm{e}^{\prime}$ at rest) and diastolic reserve index was calculated as $\Delta \mathrm{e}^{\prime} /$ rest $^{\prime}$.

\section{Endothelial function by brachial artery reactivity}

Brachial artery reactivity testing was performed to evaluate endothelial function according to standard protocols. ${ }^{11}$ In preparation for the test, participants were asked to avoid high-fat foods, smoking, vasoactive drugs, caffeine and alcohol for 24 hours, fast for 8 hours prior, and avoid strenuous exercise up to 2 hours prior to the study. Examinations took place between 8:00 and 10:00 am to avoid circadian fluctuations. Brachial artery reactivity testing on the right arm was performed in a quiet and temperature controlled room following a 
15 minute unperturbed rest with minimal conversation. In brief, the brachial artery was imaged with a $5-13 \mathrm{MHz}$ linear matrix array transducer above the antecubital fossa in the longitudinal plane. The transmit focus was placed at the anterior vessel wall with the gain and zoom settings optimised. A segment with clear definition of the anterior and posterior intimal interfaces was identified and selected for continuous 2D imaging. Anatomical landmarks such as veins and bands were noted to help maintain imaging of the same segment of the artery throughout the study. A sphygmomanometer cuff was placed on the forearm. A baseline resting image was acquired with pulsed wave Doppler examination of the flow velocity with the sample volume placed in the mid artery and aligned with the blood flow. The cuff was then inflated to $240 \mathrm{~mm} \mathrm{Hg}$ and maintained for 4.5 minutes. A 2D image during ischaemia was acquired. The cuff was then deflated and $45 \mathrm{~s}$ later, the flow velocity followed by 2D images of the brachial artery was taken during this hyperaemic phase. The participants were then rested for at least 20 minutes and until the flow velocities had returned to baseline values. A sublingual nitrate tablet $(300 \mu \mathrm{g})$ was then administered and a repeat 2D image was acquired 3 minutes following tablet dissolution.

The brachial artery diameter was measured from the intima-lumen interface of the opposing vessel walls at rest and during hyperaemia. Flow mediated dilation (FMD) was defined as the \% dilation of the vessel, measured as the difference in diameters between the hyperaemic and resting phases divided by the resting diameter. Flow rate was measured as the product of cross-sectional area of the vessel assuming a circular geometry and the time velocity integral of the antegrade flow velocities. The averages of at least three cardiac cycles were used.

\section{Statistical analysis}

Continuous variables were presented as mean $+/-1$ SD unless otherwise stated. Continuous variables were compared with the Student's t test. Categorical variables were compared with the $\chi^{2}$ test. Multiple linear regression analysis was performed to identify independent predictors of $\Delta \mathrm{e}^{\prime}$, diastolic reserve index and METs in all participants. A two-tailed $\mathrm{p}$ value $<0.05$ was considered significant. All statistical analyses were performed using STATA V.12 (STATA Corporation, College Station, Texas, USA).

\section{RESULTS}

The baseline clinical and echocardiographic characteristics of our 96 patients and 10 controls are presented in table 1. Patients and controls were well matched in the baseline clinical characteristics except for a higher prevalence of hypertension and dyslipidaemia and a higher body mass index in patients. More patients were on a statin. Patients with DM had a smaller LV end systolic volume and a low septal $\mathrm{e}^{\prime}$ at rest. Otherwise, the baseline echocardiographic characteristics were comparable. The mean HbAlc in patients with DM was $8.4 \%$.

\section{Exercise echocardiography}

Post-exercise physiological and echocardiographic parameters are presented in table 2. Compared with controls, patients had a significantly reduced exercise capacity, lower peak systolic blood pressure and lower peak heart rate. Immediately after exercise, patients with DM had lower septal $\mathrm{s}^{\prime}, \mathrm{e}^{\prime}$ and $\mathrm{a}^{\prime}$ velocities and a significantly higher septal $\mathrm{E} / \mathrm{e}^{\prime}$ ratio. Patients had a smaller $\Delta \mathrm{e}^{\prime}$ with exercise and a lower diastolic reserve index.

\section{Brachial artery reactivity}

Patients, compared with controls, had a significantly lower FMD ( 5 vs $15 \%, \mathrm{p}<0.001$ ) of the brachial artery, and a reduced relative and absolute increase in flow rate with hyperaemia (103 vs $277 \%, \mathrm{p}=0.046$ and 76 vs $189 \mathrm{~mL} / \mathrm{min}, \mathrm{p}=0.028$, respectively).

There was a significant correlation in patients with DM between FMD and $\Delta \mathrm{e}^{\prime}(\mathrm{r}=0.59, \mathrm{p}<0.001)$, diastolic reserve index $(r=0.56, p<0.001)$, post-exercise septal $\mathrm{E} / \mathrm{e}^{\prime}$ $(\mathrm{r}=-0.40, \mathrm{p}<0.001)$ and HbAlc $(\mathrm{r}=-0.21, \mathrm{p}=0.043)$, but not with resting $\mathrm{e}^{\prime}(\mathrm{r}=-0.0006, \mathrm{p}=0.996)$. After controlling for smoking, hypertension, dyslipidaemia, DM and obesity, FMD was an independent predictor of both $\Delta \mathrm{e}^{\prime}$ $\left(\beta=0.002, p<0.001, R^{2}=0.47\right)$ and diastolic reserve index $\left(\beta=0.030, p<0.001, R^{2}=0.41\right)$ in all participants.

\section{Determinants of exercise capacity}

Table 3 summarises the independent predictors of exercise capacity in all participants. Younger age, male gender, lower rest $\mathrm{E} / \mathrm{e}^{\prime}$ and higher FMD were significant independent predictors of higher exercise capacity expressed in METs $\left(\mathrm{R}^{2}=0.52, \mathrm{p}<0.001\right)$. The coefficients for age, BMI and rest $\mathrm{E} / \mathrm{e}^{\prime}$ were negative as higher values of these parameters were associated with lower METs. The coefficients for male gender and FMD were positive as male gender and higher FMD were associated with higher METs.

\section{DISCUSSION}

Patients with DM have been shown to have impaired LV diastolic function. This is also evident in the present study which demonstrated that such patients had a lower resting $\mathrm{e}^{\prime}$ despite a similar LV mass index, LV ejection fraction and $\mathrm{LV}$ filling pressures, measured by the $\mathrm{E} / \mathrm{e}^{\prime}$ ratio, compared with controls. Furthermore, these patients had impaired diastolic reserve. They were less able to augment their LV diastolic function and maintain normal LV filling pressures with exercise. This was reflected by a lower exercise capacity, lower peak rate pressure product, lower $\mathrm{e}^{\prime}$ post-exercise, less increase in $\mathrm{e}^{\prime}$, a higher $\mathrm{E} / \mathrm{e}^{\prime}$ and a lower diastolic reserve index. In agreement with other published studies ${ }^{12}$ we have also demonstrated endothelial dysfunction in our patients 
Table 1 Baseline clinical, resting echocardiographic characteristics of patients with diabetes compared with patients without diabetes

\begin{tabular}{|c|c|c|c|}
\hline & Diabetes $(n=96)$ & No diabetes $(n=10)$ & p Value \\
\hline \multicolumn{4}{|l|}{ Clinical and anthropological characteristics } \\
\hline Age (years) & $56 \pm 9$ & $50 \pm 7$ & 0.058 \\
\hline Male & $58(60 \%)$ & $7(70 \%)$ & 0.554 \\
\hline Height $(\mathrm{cm})$ & $168 \pm 9$ & $172 \pm 11$ & 0.127 \\
\hline Weight $(\mathrm{kg})$ & $94 \pm 25$ & $83 \pm 18$ & 0.148 \\
\hline Body surface area $\left(\mathrm{m}^{2}\right)$ & $2.08 \pm 0.29$ & $1.98 \pm 0.276$ & 0.304 \\
\hline Body mass index $\left(\mathrm{kg} / \mathrm{m}^{2}\right)$ & $34 \pm 8$ & $28 \pm 4$ & 0.025 \\
\hline Smoking n (\%) & $13(14 \%)$ & $1(10 \%)$ & 0.753 \\
\hline Hypertension $\mathrm{n}(\%)$ & $71(74 \%)$ & $2(20 \%)$ & $<0.001$ \\
\hline Dyslipidaemia n (\%) & 77 (80\%) & $4(40 \%)$ & 0.004 \\
\hline Family history CAD n (\%) & 31 (32\%) & $4(40 \%)$ & 0.622 \\
\hline Antiplatelet agent $\mathrm{n}(\%)$ & $33(34 \%)$ & $1(10 \%)$ & 0.116 \\
\hline$\beta$-blocker n (\%) & $10(10 \%)$ & $0(0 \%)$ & 0.593 \\
\hline Calcium channel blocker $\mathrm{n}(\%)$ & $21(22 \%)$ & $0(0 \%)$ & 0.205 \\
\hline ACE inhibitor $n(\%)$ & $38(40 \%)$ & $0(0 \%)$ & 0.013 \\
\hline Angiotensin receptor blocker n (\%) & $34(35 \%)$ & $1(10 \%)$ & 0.160 \\
\hline Diuretics n (\%) & $18(19 \%)$ & $0(0 \%)$ & 0.206 \\
\hline Statin $n(\%)$ & $55(57 \%)$ & $2(20 \%)$ & 0.042 \\
\hline Fenofibrate & $2(2 \%)$ & $0(0 \%)$ & 1.000 \\
\hline MRC dyspnoea grade & & & 0.397 \\
\hline 1 & $30(31 \%)$ & $6(60 \%)$ & \\
\hline 2 & $50(52 \%)$ & $4(40 \%)$ & \\
\hline 3 & $8(8 \%)$ & $0(0 \%)$ & \\
\hline 4 & $8(8 \%)$ & $0(0 \%)$ & \\
\hline 5 & $0(0 \%)$ & $0(0 \%)$ & \\
\hline \multicolumn{4}{|l|}{ Resting left ventricular parameters } \\
\hline $\mathrm{LV}$ end-diastolic volume index $\left(\mathrm{mL} / \mathrm{m}^{2}\right)$ & $35 \pm 26$ & $45 \pm 13$ & 0.09 \\
\hline LV end-systolic volume index $\left(\mathrm{mL} / \mathrm{m}^{2}\right)^{\prime}$ & $10 \pm 6$ & $15 \pm 6$ & 0.021 \\
\hline Resting LV ejection fraction (\%) & $70 \pm 11$ & $66 \pm 6$ & 0.116 \\
\hline Septal $\mathrm{s}^{\prime}(\mathrm{cm} / \mathrm{s})$ & $7.0 \pm 1.4$ & $7.3 \pm 1.3$ & 0.463 \\
\hline Septal e' (cm/s) & $6.7 \pm 1.7$ & $8.6 \pm 2.0$ & 0.002 \\
\hline Septal E/e & $10.2 \pm 4.2$ & $7.7 \pm 1.5$ & $<0.001$ \\
\hline Lateral s' (cm/s) & $7.6 \pm 2.1$ & $7.3 \pm 2.0$ & 0.687 \\
\hline Lateral $e^{\prime}(\mathrm{cm} / \mathrm{s})$ & $8.2 \pm 2.4$ & $9.7 \pm 3.5$ & 0.092 \\
\hline Lateral E/e' & $8.6 \pm 4.0$ & $7.9 \pm 5.1$ & 0.593 \\
\hline LV mass index $\left(\mathrm{g} / \mathrm{m}^{2}\right)$ & $76 \pm 20$ & $75 \pm 18$ & 0.910 \\
\hline
\end{tabular}

Table 2 Post-exercise echocardiographic parameters and exercise physiological responses of patients with diabetes mellitus compared to those without diabetes

\begin{tabular}{|c|c|c|c|}
\hline & Diabetes $(n=96)$ & No diabetes $(n=10)$ & p Value \\
\hline \multicolumn{4}{|l|}{ Post-exercise echocardiographic parameters } \\
\hline LV end-diastolic volume index (mL/m²) & $36 \pm 12$ & $46 \pm 12$ & 0.015 \\
\hline LV end-systolic volume index $\left(\mathrm{mL} / \mathrm{m}^{2}\right)$ & $10 \pm 7$ & $10 \pm 5$ & 0.716 \\
\hline LV ejection fraction (\%) & $74 \pm 10$ & $78 \pm 8$ & 0.252 \\
\hline Septal s' (cm/s) & $10.9 \pm 2.8$ & $14.6 \pm 4.6$ & 0.033 \\
\hline Septal e' (cm/s) & $7.9 \pm 2.1$ & $12.5 \pm 4.1$ & 0.006 \\
\hline Septal E/e' & $12.3 \pm 4.0$ & $7.0 \pm 1.9$ & $<0.001$ \\
\hline$\Delta \mathrm{e}^{\prime}(\mathrm{cm} / \mathrm{s})$ & $1.2 \pm 1.5$ & $3.9 \pm 3.2$ & 0.023 \\
\hline$\Delta \mathrm{e}^{\prime} /$ resting $\mathrm{e}^{\prime}$ & $0.20 \pm 0.26$ & $0.47 \pm 0.34$ & 0.003 \\
\hline \multicolumn{4}{|l|}{ Exercise parameters } \\
\hline METs & $8.5 \pm 2.8$ & $13.2 \pm 2.9$ & $<0.001$ \\
\hline Peak heart rate (bpm) & $152 \pm 19$ & $168 \pm 13$ & 0.012 \\
\hline Peak systolic blood pressure $(\mathrm{mm} \mathrm{Hg})$ & $168 \pm 19$ & $184 \pm 16$ & 0.018 \\
\hline Peak diastolic blood pressure $(\mathrm{mm} \mathrm{Hg})$ & $70 \pm 11$ & $73 \pm 11$ & 0.471 \\
\hline
\end{tabular}

LV, left ventricular; METs, metabolic equivalents. 
Table 3 Independent determinants of exercise capacity measured as metabolic equivalents in all participants

\begin{tabular}{lccc}
\hline Parameter & Coefficient & $\mathbf{9 5 \%} \mathbf{C l}$ & p Value \\
\hline Age & -0.137 & -0.19 to -0.08 & $<0.001$ \\
Male gender & 1.19 & 0.24 to 2.13 & 0.014 \\
BMI & -0.176 & -0.24 to -0.12 & $<0.001$ \\
Septal E/e' at rest & -0.149 & -0.29 to -0.005 & 0.04 \\
FMD & 0.092 & 0.01 to 0.17 & 0.025 \\
Constant & 22.86 & 18.9 to 26.8 & $<0.001$ \\
\hline BMl, body mass index; FMD, flow-mediated dilation.
\end{tabular}

with DM manifested as impaired FMD of the brachial artery. More importantly, we have shown that there was a significant relationship between endothelial function, exercise capacity and LV diastolic reserve. After correcting for factors known to affect diastolic function and exercise capacity, endothelial function remained an independent predictor of diastolic reserve and exercise capacity.

\section{Diastolic dysfunction in diabetes}

Patients with diabetes are at risk of developing heart failure independent of traditional risk factors like hypertension or CAD with subclinical LV systolic and diastolic dysfunction common even in asymptomatic patients. An impaired diastolic reserve in DM may even be an earlier manifestation of LV involvement, at a stage where the resting systolic and diastolic function may still be within normal range. Ha et al demonstrated a blunted increase in the mitral annular $\mathrm{s}^{\prime}$ and $\mathrm{e}^{\prime}$ velocities in patients with type $2 \mathrm{DM}$ compared with controls despite similar velocities between the two groups at rest. ${ }^{13}$ A similar impairment in increase in $\mathrm{e}^{\prime}$ velocities was noted by Jellis $e t$ al in patients with DM and normal resting $\mathrm{e}^{\prime}$ velocities. ${ }^{14}$ In the current study, we were able to demonstrate that patients with type $2 \mathrm{DM}$ had worse LV diastolic function and elevated diastolic pressures post-exercise. Impaired diastolic reserve is also of prognostic significance in DM. In a study of 197 patients with DM followed up over a median of 57 months, eGFR, DM duration and the increase in $\mathrm{e}^{\prime}$ with exercise were found to be independently predictive of adverse cardiac outcome. ${ }^{15}$ Diabetic cardiomyopathy is believed by many to be a distinct clinical entity with multiple mechanisms, ${ }^{16}{ }^{17}$ with microvascular dysfunction as the final common pathway in the pathogenesis. Previous studies have suggested a possible link between microvascular function and LV diastolic function, ${ }^{18}$ but the correlation has not been examined previously.

\section{Endothelial and diastolic dysfunction in diabetes}

FMD of large conduit vessels is an accepted technique to quantify endothelial function. It has been found to have predictive value for future cardiovascular events. ${ }^{19}$ Furthermore, as endothelial dysfunction is a systemic process, endothelial function in the peripheral circulation is used as a surrogate of coronary microvascular function, with good correlation demonstrated between responses to acetylcholine in the coronary circulation and in the forearm vessels. ${ }^{20}$ Endothelial dysfunction has been demonstrated in patients with DM. ${ }^{12}$ We have shown that endothelial function, diastolic reserve and exercise capacity are intricately linked with the endothelial function being a significant independent determinant of both diastolic reserve and exercise capacity. It can be inferred that, among other known factors including age and gender that affect exercise capacity, the ability of the left ventricle to augment diastolic function is determined by coronary microvascular function which ultimately determines exercise capacity. $\mathrm{LV}$ diastole is an active energy requiring process and we have been able to demonstrate that diastolic dysfunction, seen in disease states like DM, is mediated by coronary microvascular dysfunction. Measures that are known to improve endothelial function, such as treatment of hypertension, dyslipidaemia and cessation of smoking, may help to improve LV diastolic function and exercise capacity.

\section{CONCLUSIONS}

Patients with DM had impaired endothelial function and LV diastolic dysfunction. LV diastolic functional reserve and exercise capacity are linked to endothelial function. LV diastolic functional reserve in DM is mediated by endothelial function, in addition to other known pathogenetic factors of diabetic cardiomyopathy. Targeting vascular risk factors that impair endothelial function may improve LV diastolic reserve and exercise capacity.

Contributors ML jointly conceived the study with DYL, designed and implemented the trial, and obtained ethics approval; ML, DYL and VP performed the literature review and were involved in the recruitment of subjects, testing of trial patients, data collection and preparation of the manuscript.

Funding $M L$ was funded by a postgraduate scholarship from the National Health and Medical Research Council of Australia.

\section{Competing interests None.}

Ethics approval Concord hospital human research ethics committee.

Provenance and peer review Not commissioned; externally peer reviewed.

Data sharing statement No additional data are available.

Open Access This is an Open Access article distributed in accordance with the Creative Commons Attribution Non Commercial (CC BY-NC 3.0) license, which permits others to distribute, remix, adapt, build upon this work noncommercially, and license their derivative works on different terms, provided the original work is properly cited and the use is non-commercial. See: http:// creativecommons.org/licenses/by-nc/3.0/

\section{REFERENCES}

1. Epstein $\mathrm{FH}$, Ross R. Atherosclerosis-an inflammatory disease. N Engl J Med 1999;340:115-26.

2. Mano T, Masuyama T, Yamamoto $\mathrm{K}$, et al. Endothelial dysfunction in the early stage of atherosclerosis precedes appearance of intimal lesions assessable with intravascular ultrasound. Am Heart $J$ 1996;131:231-8. 
3. Widlansky ME, Gokce N, Keaney JF, et al. The clinical implications of endothelial dysfunction. J Am Coll Cardiol 2003;42:1149-60.

4. Cai $\mathrm{H}$, Harrison DG. Endothelial dysfunction in cardiovascular diseases: the role of oxidant stress. Circ Res 2000;87:840-4.

5. Borlaug BA, Olson TP, Lam CSP, et al. Global cardiovascular reserve dysfunction in heart failure with preserved ejection fraction. J Am Coll Cardiol 2010;56:845-54.

6. Ha JW, Oh JK, Pellikka PA, et al. Diastolic stress echocardiography: a novel noninvasive diagnostic test for diastolic dysfunction using supine bicycle exercise Doppler echocardiography. J Am Soc Echocardiogr 2005;18:63-8.

7. Holland DJ, Prasad SB, Marwick TH. Contribution of exercise echocardiography to the diagnosis of heart failure with preserved ejection fraction (HFpEF). Heart 2010;96:1024.

8. Association AD. Diagnosis and classification of diabetes mellitus. Diabetes Care 2007;30(suppl 1):S42-7.

9. Levey AS, Bosch JP, Lewis JB, et al. A more accurate method to estimate glomerular filtration rate from serum creatinine: a new prediction equation. Ann Intern Med 1999;130:461-70.

10. Armstrong $\mathrm{L}$. ACSM's guidelines for exercise testing and prescription/American College of Sports Medicine. Philadelphia, PA: Lippincott Williams \& Wilkins, 2006.

11. Corretti MC, Anderson TJ, Benjamin EJ, et al. Guidelines for the ultrasound assessment of endothelial-dependent flow-mediated vasodilation of the brachial artery: a report of the International Brachial Artery Reactivity Task Force. J Am Coll Cardiol 2002;39:257-65.

12. Mäkimattila S, Liu M-L, Vakkilainen J, et al. Impaired endothelium-dependent vasodilation in type 2 diabetes. Relation to
LDL size, oxidized LDL, and antioxidants. Diabetes Care 1999;22:973-81.

13. Ha J-W, Lee H-C, Kang E-S, et al. Abnormal left ventricular longitudinal functional reserve in patients with diabetes mellitus: implication for detecting subclinical myocardial dysfunction using exercise tissue Doppler echocardiography. Heart 2007; 93:1571-6.

14. Jellis CL, Stanton T, Leano R, et al. Usefulness of at rest and exercise hemodynamics to detect subclinical myocardial disease in type 2 diabetes mellitus. Am J Cardiol 2011;107:615-21.

15. Kim SA, Shim CY, Kim JM, et al. Impact of left ventricular Iongitudinal diastolic functional reserve on clinical outcome in patients with type 2 diabetes mellitus. Heart 2011;97:1233.

16. Boudina S, Abel ED. Diabetic cardiomyopathy, causes and effects. Rev Endocr Metab Disord 2010;11:31-9.

17. Galderisi M. Diastolic dysfunction and diabetic cardiomyopathy: evaluation by Doppler echocardiography. J Am Coll Cardio 2006:48:1548-51.

18. Pop-Busui R, Kirkwood I, Schmid H, et al. Sympathetic dysfunction in type 1 diabetes: association with impaired myocardial blood flow reserve and diastolic dysfunction. J Am Coll Cardiol 2004;44: 2368-74.

19. Ras RT, Streppel MT, Draijer R, et al. Flow-mediated dilation and cardiovascular risk prediction: a systematic review with meta-analysis. Int J Cardiol 2013;168:344-51.

20. Anderson TJ, Uehata A, Gerhard MD, et al. Close relation of endothelial function in the human coronary and periphera circulations. J Am Coll Cardiol 1995;26:1235-41. 